UVX 2008 (2009) 133-138

(C) EDP Sciences, 2009

DOI: $10.1051 / \mathrm{uvx} / 2009021$

\title{
Étude théorique de la photoionisation à deux couleurs XFEL-IR
}

\author{
R. Taïeb ${ }^{1}$, A. Maquet ${ }^{1}$ et M. Meyer ${ }^{2}$ \\ ${ }^{1}$ UPMC, Université Paris 06, CNRS, UMR 7614, Laboratoire de Chimie Physique-Matière \\ et Rayonnement, 11 rue Pierre et Marie Curie, 75231 Paris Cedex 05, France \\ ${ }^{2}$ LIXAM, UMR 8624, Université Paris Sud, Bâtiment 350, 91405 Orsay Cedex, France
}

\begin{abstract}
Résumé. Nous aborsdons plusieurs questions liées aux processus non linéaires observés quand des atomes sont en présence simultanée d'impulsions laser intenses et cohérentes infrarouge (IR) et ultraviolet lointain (XUV). L'essor de ce sujet est très fortement lié au développement de nouvelles sources cohérentes XUV, soit à base des harmoniques d'ordre élevé ou des laser à électrons libres (FEL), comme celui disponible au FLASH à Hambourg. Ces sources peuvent être synchronisées avec des laser IR. Dans cet article, nous discutons aussi les avantages et les limitations de l'approximation dite "soft-photon", qui, comme nous le montrerons ici, fournit des informations extrêmement utiles dans l'analyse des différents processus impliquant ces deux champs.
\end{abstract}

\section{INTRODUCTION}

L'ionisation multiphotonique à "une couleur" dans les domaines optique ou infrarouge en champ intense a fait l'objet de multiples études tant expérimentales que théoriques pendant plus de deux décennies et est à ce jour un processus très bien compris (voir par exemple [1]). Le prolongement de ces études à l'absorption multiphotonique dans le régime XUV devient maintenant possible avec l'arrivée de nouvelles sources de rayonnement intenses comme la génération d'harmoniques d'ordres élevés (HHG) d'un champ d'IR ou le laser à électrons libres à Hambourg (FLASH). Ce dernier, fondé sur le processus de l'émission spontanée auto-amplifiée (SASE), est une source de lumière laser cohérente extrêmement intense et ultra-courte (environ $20 \mathrm{fs}$ ). Par conséquent, les équipements comportant des impulsions infrarouges (IR) synchronisées avec un laser ultraviolet, rendent faisable le développement de nouveaux outils de spectroscopie pour étudier les propriétés du continuum atomique et/ou moléculaire. Dans cet article, nous arguerons du fait que, dans ce contexte, l'approximation dite "soft-photon" fournit un cadre conceptuel très commode pour relever les défis soulevés par les résultats obtenus à partir d'un tel nouvel ensemble d'expériences. Nous présenterons les différents schémas qui fourniront des informations utiles sur la dynamique de ces processus à "deux couleurs".

\section{THÉORIE}

Le développement des sources laser IR intenses, ces 40 dernières années, a fourni aux expérimentateurs et théoriciens un outil utile afin d'explorer différents processus multiphotoniques dans les atomes ou les petites molécules. Dans la figure 1, nous résumons ces processus allant de l'ionisation multiphotonique observée vers la fin des annés 60, à l'ionisation “au-dessus du seuil" (ATI), où l'électron absorbe plus de photons IR que nécessaire pour être ionisé et à la génération d'harmoniques d'ordre élevé. L'arrivée des sources intenses XUV pose la question de la transposition de certains de ces processus à des énergies de photons plus élevées. Afin d'y répondre, on peut considérer le mouvement d'un électron classique dans un champ électrique $F_{0}$, de fréquence $\omega_{L}$, polarisé le long de l'axe des abscisses $\epsilon / /(O x)$. L'équation de Newton s'écrit alors comme :

$$
m \frac{d^{2} \mathbf{x}}{d t^{2}}=q F_{0} \epsilon \sin \left(\omega_{L} t+\phi\right)
$$




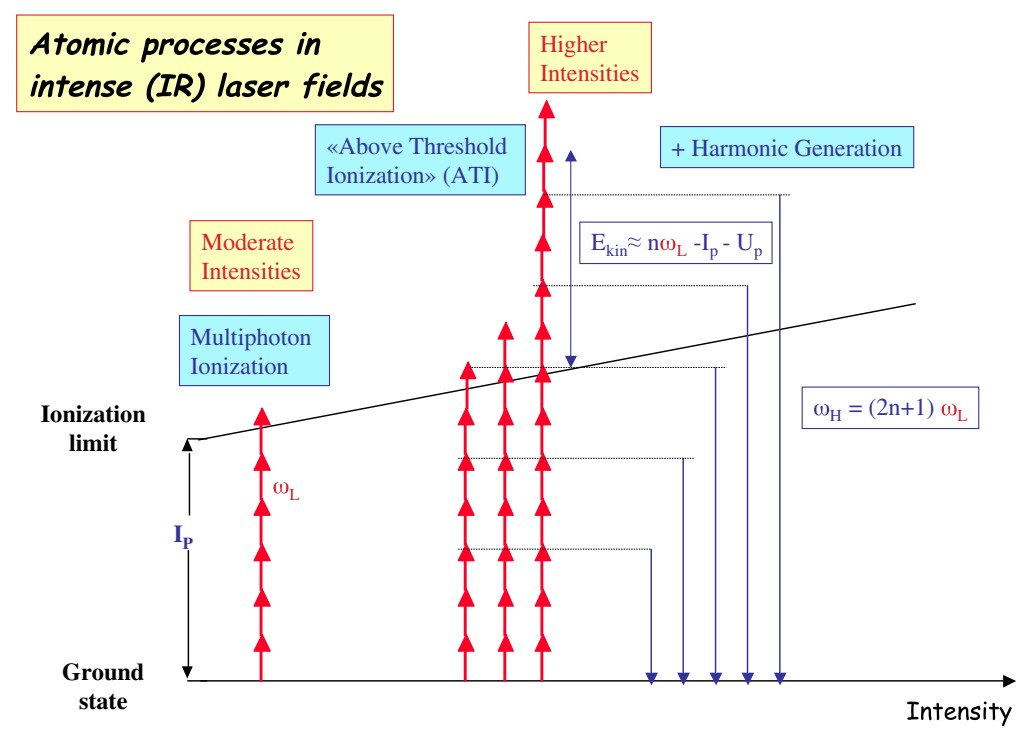

Figure 1. Processus observables pour un laser IR en fonction de l'intensité du champ. $I_{P}$ est le potentiel d'ionisation, $\omega_{L}$ la fréquence du laser et $U_{p}$ l'énergie ponderomotive, cf. Eq. (5).

En intégrant cette équation, nous obtenons les expressions pour la vitesse et la position de l'électron sous la forme :

$$
\begin{aligned}
\mathbf{v}(t)=\frac{d \mathbf{x}}{d t} & =-\frac{q F_{0} \epsilon}{m \omega_{L}}\left[\cos \left(\omega_{L} t+\phi\right)-\cos (\phi)\right]+\mathbf{v}(0) \\
\mathbf{x}(t) & =-\alpha_{0}\left[\sin \left(\omega_{L} t+\phi\right)-\sin (\phi)\right]+\left[\mathbf{v}(0)+\alpha_{0} \omega_{L} \cos (\phi)\right] t+\mathbf{x}(0) . \\
\alpha_{0} & =\frac{q F_{0} \epsilon}{m \omega_{L}^{2}} .
\end{aligned}
$$

Ici, $\mathbf{v}(0), \mathbf{x}(0)$ sont respectivement la vitesse et la position initiale et $\alpha_{0}$ l'amplitude du mouvement oscillant. Dans le cas où l'électron s'ionise par effet tunnel, ces deux grandeurs sont égales à 0 $(v(0)=x(0)=0)$. Cependant, si nous considérons que l'ionisation est due à l'absorption d'un photon XUV de fréquence $\omega_{X}$, nous avons $v^{2}(0) /(2 m)=\omega_{X}-I_{p}$ où $I_{P}$ est l'énergie d'ionisation de l'atome.

En moyennant sur le temps l'énergie cinétique du mouvement oscillant, on reconnaît l'énergie ponderomotrice donnée par :

$$
\frac{1}{2 m}\left\langle v^{2}(t)\right\rangle=U_{p}=\frac{q^{2} F_{0}^{2}}{4 m \omega_{L}^{2}} .
$$

Un traitement semblable donne la position de la coupure des spectres de la génération d'harmoniques d'ordres élevés [2]. On notera la dépendance en $\omega_{L}^{-2}$ pour les deux grandeurs $\alpha_{0}$ et $U_{p}$. Par conséquent ces deux quantités, qui deviennent comparables aux quantités atomiques $\left(a_{0} \approx 0.529 \AA\right.$, le rayon de Bohr, et $I_{P} \approx 10 \mathrm{eV}$, l'énergie d'ionisation) pour une intensité $\approx 10^{14} \mathrm{~W} \mathrm{~cm}^{-2}$ d'un laser IR (Ti :Sapphire) de fréquence $\omega_{L} \approx 1.55 \mathrm{eV}$, deviennent negligeables pour un champ de même intensité dans le domaine XUV de fréquence $\approx 92 \mathrm{eV}$ (comme celle de FLASH). On peut donc conclure que les processus multiphotoniques seront beaucoup plus difficilement observables dans le domaine XUV que dans l'infrarouge. Cependant, il faut noter que de toutes nouvelles expériences réaliséss à FLASH par le groupe de M. Richter ont pu observer l'absorption de plus d'une cinquantaine de photons de $92 \mathrm{eV}$ par un atome de xénon pour obtenir l'ion $X e^{21+}[3]$. 
Pour traiter la photoionisation de He résultant de l'absorption d'un photon XUV en présence d'un champ IR, on peut écrire l'amplitude de transition de matrice $S$, entre l'état fondamental de l'Helium et un état de final du continuum "habillé" par le laser IR, [4] :

$$
S=-i \int_{-\infty}^{+\infty} d r\left\langle\chi_{\mathbf{k}}(\mathbf{r}, t)\left|\frac{1}{c} \mathbf{A}_{X} \cdot \mathbf{p}\right| \psi_{1 s^{2}}(\mathbf{r}, t)\right\rangle .
$$

où $\mathbf{A}$ est le potentiel vecteur associé au champ électrique XUV, donné par $\mathbf{F}_{X}(t)=-\frac{1}{c} \frac{\partial \mathbf{A}_{X}}{\partial t}(t)$.

$$
\chi_{\mathbf{k}}(\mathbf{r}, t)=(2 \pi)^{-3 / 2} \exp \left[i\left(\mathbf{k} \cdot \mathbf{r}-\frac{k^{2}}{2} t-\alpha_{0} \cdot \mathbf{k} \sin \left(\omega_{L} t+\phi\right)\right],\right.
$$

est la fonction d'onde dite "de Volkov" représentant un électron libre dans le continuum "habillé" par le champ laser, [5]. En intégrant sur le temps et profitant des propriétés des fonctions de Bessel [6], on obtient :

$$
S=\sum_{n=-\infty}^{n=+\infty} S^{(n)} \delta\left(\frac{k^{2}}{2}-\left(E_{1 s^{2}}+\omega_{X}+n \omega_{L}\right)\right),
$$

où les composants $S^{(n)}$ sont associés à l'échange net de $n$ photons laser en plus de l'absorption d'un photon XUV. La section efficace différentielle correspondante s'écrit alors sous la forme :

$$
\frac{d \sigma^{(n)}}{d \Omega}=J_{n}^{2}\left(\alpha_{0} \cdot \mathbf{k}_{n}\right) \frac{d \bar{\sigma}}{d \Omega},
$$

où $k_{n}=\sqrt{2\left(E_{1 s^{2}}+\omega_{X}+n \omega_{L}\right)}$ désigne le vecteur d'onde de l'électron éjecté et $d \bar{\sigma} / d \Omega$, la section efficace différentielle correspondant à l'absorption d'un photon $\omega_{X}$. La section efficace totale $\sigma^{(n)}$ associée à cet échange de $n$ photons laser est obtenue par intégration sur l'angle solide $\Omega \equiv(\eta, \phi)$. Pour un électron $1 s$ de He, et après intégration sur $\phi$, nous obtenons

$$
\sigma^{(n)} \propto a_{n}+b_{n} \cos ^{2} \theta
$$

où les coefficients $a_{n}$ et $b_{n}$ sont donnés par :

$$
\left\{\begin{array}{l}
a_{n} \\
b_{n}
\end{array}=\int_{0}^{\pi} d \eta \sin \eta J_{n}^{2}\left(\alpha_{0} k_{n} \cos \eta\right)\left\{\begin{array}{l}
\sin ^{2} \eta \\
\left(2-3 \sin ^{2} \eta\right)
\end{array},\right.\right.
$$

et $\theta$ est l'angle entre les directions de polarisation des deux champs, Dans le cas de polarisations parallèles $(\theta=0)$, nous avons aussi résolu l'Équation de Schrödinger Dépendante du Temps (ESDT) afin de vérifier le domaine de validité de cette approximation "soft-photon".

\section{RÉSULTATS}

Nous présentons, Figs. 2, deux spectres théoriques de photoélectrons provenant de l'ionisation à “deux couleurs" de l'hélium. Dans ces simulations, nous avons considéré des impulsions XUV $\left(\omega_{X}=92 \mathrm{eV}\right)$ et IR $\left(\omega_{L}=1.55 \mathrm{eV}\right)$, de même durée et polarisées linéairement, avec des polarisations parallèles. Les intensités maximales respectives des champs sont $I_{X}=10^{12} \mathrm{~W} \mathrm{~cm}^{-2}$ and $I_{L}=5 \times 10^{11} \mathrm{~W} \mathrm{~cm}^{-2}$ (Fig. 2(a)) et $I_{L}=2 \times 10^{12} \mathrm{~W} \mathrm{~cm}^{-2}$ (Fig. 2(b)). Ces paramètres sont représentatifs de ceux qui sont actuellement employés dans les expériences à FLASH. En l'absence du laser, on observe un seul pic de photoélectron située à $k_{0}^{2} / 2 \approx 68 \mathrm{eV}$, cf Fig. 2(a). Nous mentionons que l'absorption de 2 photons XUV de $92 \mathrm{eV}$ est complètement négligeable à cette intensité.

Dans le cas de l'ionisation à "deux couleurs", le spectre de photoélectron se compose d'un pic principal lié à l'absorption d'un photon XUV accompagné de pics latéraux, placés symétriquement de chaque côté, cf. Figs. 2. Ces pics satellites séparés d'une même quantité $\Delta E=\omega_{L}$, sont associés à l'échange additionnel de photons du laser IR, par des processus d'absorption et/ou d'émission stimulée. 

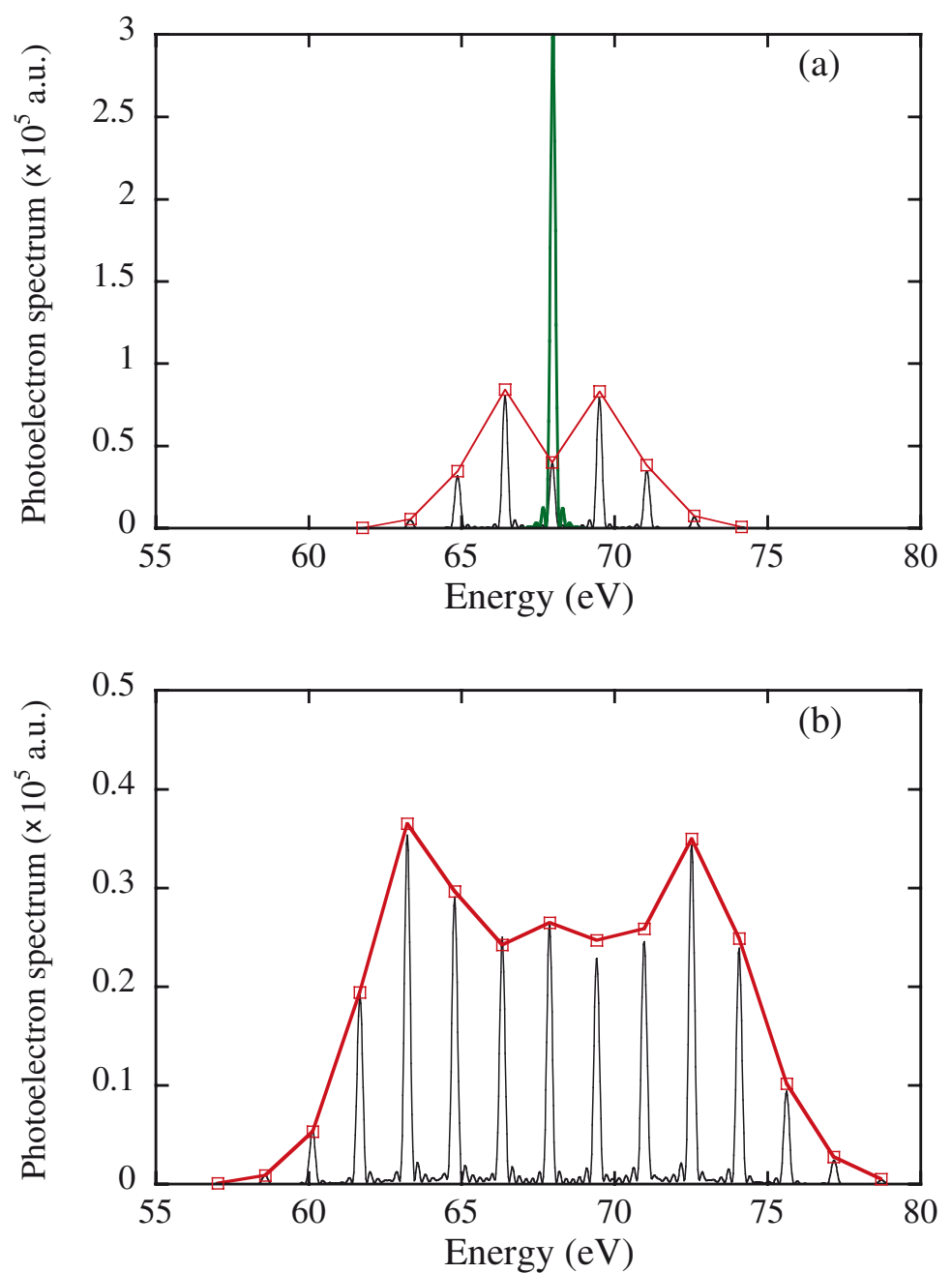

Figure 2. Spectres de photoélectrons correspondant à la photoionisation de $\mathrm{He}$ par "2-couleurs" composées d'un champ IR $\left(\omega_{L}=1.55 \mathrm{eV}\right)$ et d'un champ XUV $\left(\omega_{X}=92 \mathrm{eV}, I_{X}=10^{12} \mathrm{~W} \mathrm{~cm}^{-2}\right)$. Les deux champs ont des polarisations parallèles. Trait noir fin : Calcul TDSE ; carrés rouges : approximation "soft-photon". (a) $I_{L}=5 \times 10^{11} \mathrm{~W} \mathrm{~cm}^{-2}$. Trait Vert épais correspond au spectre d'ionisation suite à l'absorption d'un photon XUV en l'absence d'IR. (b) $I_{L}=2 \times 10^{12} \mathrm{~W} \mathrm{~cm}^{-2}$.

On note l'excellent accord entre nos deux approches théoriques, donnant confiance quant à la validité de l'approximation "soft-photon", à la fois sur le plan qualitatif mais aussi quantitatif. De plus, nous voyons que le nombre et la magnitude des pics satellites dépendent fortement de l'intensité du champ IR et du recouvrement spatial et temporel entre les deux champs. Nous avons utilisé ce fait pour mesurer le "jitter" temporel du FEL [7,8]. En outre, nous avons récemment réalisé des expériences sur FLASH, où nous avons varié l'angle entre les polarisations des deux champs, cf. Figs. 3 [10]. Expérimentalement, les champs laser XUV FEL et IR sont introduits dans une chambre "ultra-vide" avec une géométrie colinéaire et intersectant un jet de gaz effusif dans le volume d'acceptance d'un spectromètre d'électrons formé d'une bouteille magnétique. Afin de s'assurer du recouvrement temporel parfait entre les impulsions et pour éliminer les effets inhérents au "jitter" du FEL $\approx 500$ fs [7], les expériences ont été réalisées avec des lasers IR picosecondes synchronisés aux impulsions FEL de 

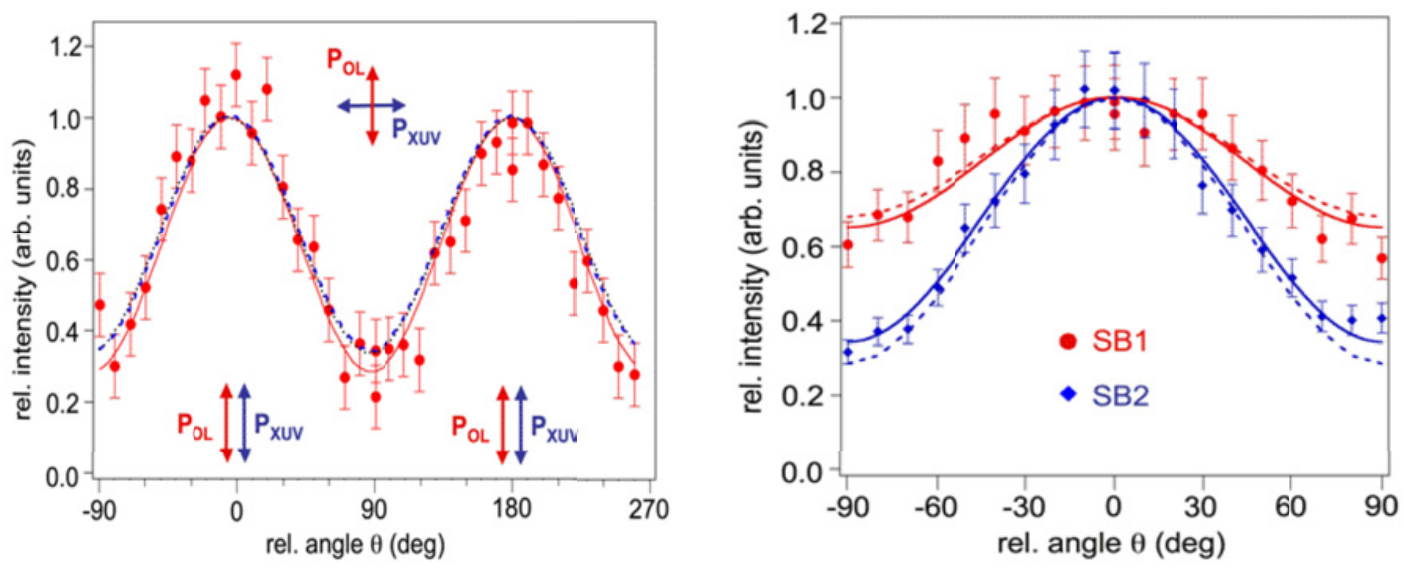

Figure 3. Variation des pics satellites correspondant à l'ionisation de He par absorption d'un photon XUV $\left(\omega_{X}=\right.$ $92 \mathrm{eV}$ ) et d'un (SB1) ou deux (SB2) photons IR $\left(\omega_{L}=1.55 \mathrm{eV}\right)$ en fonction de l'angle relatif entre les vecteurs de polarisation du FEL et IR. Panneau gauche : régime basse intensité $\left(I_{L}=8 \times 10^{10} \mathrm{~W} \mathrm{~cm}^{-2}\right)$. Panneau droit : Régime d'intensité modéré $\left(I_{L}=6 \times 10^{11} \mathrm{~W} \mathrm{~cm}^{-2}\right)$. les lignes continues dénotent les données expérimentales (cercles et diamants pour le premièr et deuxième pic satellite, respectivement). Les lignes pointillées représentent les résultats de l'approximation "soft-photon".

10-20fs [9]. La polarisation respectives des deux lasers était presque parfaitement linéaire et l'orientation relative entre les vecteurs de polarisation a pu être changée par une lame demi-onde rotative placée sur le chemin optique du laser IR.

On voit Fig. 3 que l'intensité du premier pic satellite (noté SB1) varie avec l'angle entre les polarisations suivant une loi en $\cos ^{2}$. On peut relier le contraste de ces variations avec le poids des différentes ondes partielles présentes dans le processus [10]. De plus, en comparant ce contraste à basse (gauche) et haute (droite) intensité du laser IR, on s'aperçoit qu'il diminue fortement lorsque l'intensité IR croît. Nous allons ci-dessous expliquer les raisons d'un tel comportement grâce à l'approximation "soft-photon". A basse intensité du laser IR, c.-à-d. $z=\alpha_{0} k_{n}<<1$, cf. Fig. 3 (gauche), la fonction de Bessel dans l'Eq. (9) est proportionnelle à $\left(\alpha_{0} k_{n} \cos \eta\right)^{n}$, ce qui donne $b_{n} / a_{n}=2 n$, d'où un contraste de $\sigma_{\text {max }}^{(n)} / \sigma_{\text {min }}^{(n)}=2 n+1$ dans la dépendance angulaire de la section efficace $\sigma^{(n)}$. Ceci coïncide avec le résultat à basse intensité où nous obtenons un contraste expérimental de $3.25 \pm 0.25$ très proche de la valeur théorique de $3(n=1)$. Cependant, à plus haute intensité, cf. Fig. 3 (droite), $z \approx 1$, on obtient pour le premier pic satellite $(n=1)$. Ceci montre que le rapport $b_{1} / a_{1}$ est réduit lorque l'intensité du laser IR augmente, réduisant d'autant le contraste dans la distribution angulaire. Ceci rappelle ce qui a été observé en combinant des harmoniques XUV avec un laser d'IR: la contribution des moments angulaires plus élevés a comme conséquence la diminution des maxima relatifs des distributions angulaires [11].

Ce contraste réduit dans la distribution angulaire peut être expliqué en exécutant a développement de la fonction Bessel

$$
\begin{aligned}
& a_{1} \approx \frac{z^{2}}{15}\left[1-\frac{3 z^{2}}{28}+\frac{25 z^{4}}{4032}\right] \\
& b_{1} \approx \frac{2 z^{2}}{15}\left[1-\frac{6 z^{2}}{28}+\frac{75 z^{4}}{4032}\right] .
\end{aligned}
$$

Pour une plus grande valeur de son $\operatorname{argument} z$. Nous pouvons, donc, prouver que le rapport $b_{1} / a_{1}$ devient plus petit avec l'augmentation de l'intensité de laser. Ceci vient du ce, l'inclusion de différentes voies, avec des moments angulaires plus élevés pour l'électron sortant, menant à la formation des premiers résultats de pic satellite dans l'interférence destructive quand les deux champs sont parallèles. 
D'ailleurs, le contraste pour le deuxième pic satellite (SB2) est, comme prévu par l'approximation "softphoton", plus grande (3.3 dans l'expérience et 3.5 dans la théorie), bien qu'il n'atteigne pas la valeur limite de $2 n+1=5$.

\section{CONCLUSION}

Nous avons étudié la validité de l'approximation "soft-photon" pour décrire les spectres de photoionisation à deux couleurs. A cet effet, nous avons comparé les résultats de la résolution numérique de la TDSE aux prévisions de cette approximation basée sur l'utilisation des fonctions d'onde de Volkov afin de décrire l'électron éjecté. Les résultats présentés ici démontrent clairement l'utilité de cette approximation dans la perspective d'interpréter les données pour des processus d'ionisation IR + XUV dans le régime de champ fort.

\section{Références}

[1] L. F. Di Mauro and P. Agostini, Adv. At. Mol. Opt. Phys. 35, 79 (1995).

[2] P. B. Corkum, Phys. Rev. Lett. 71, 1994 (1993).

[3] A. A. Sorokin et al., Phys. Rev. Lett. 99, 213002 (2007).

[4] A. Maquet and R. Taïeb, J. Mod. Opt. 54, 1847 (2007).

[5] F.V. Bunkin and M.V. Fedorov, Sov. Phys.-JETP 22844 (1966).

[6] A. Abramowitz and I.A. Stegun, Handbook of Mathematical Functions (NBS, Washington, USA, 1964).

[7] Voir, par exemple: V. Véniard, R. Taïeb, and A. Maquet, Phys. Rev. A 54, 721 (1996) et les références incluses.

[8] M. Meyer et al., Phys. Phys. A 74, 011401(R) (2006).

[9] P. Radcliffe et al., Appl. Phys. Lett. 90, 131108 (2007).

[10] M. Meyer et al., Phys. Rev. Lett. 90, 193002 (2008).

[11] O. Guyétand et al., J. Phys. B 41, 051002 (2008). 